(Aus der Deutschen Dermatologischen Klinik in Prag [Vorstand Prof. C. Kreibich].)

\title{
Carcinomentwicklung in einem Dermoid der Haut.
}

\author{
Von \\ Dr. Siegm. Schoenhof, \\ Assistent der Klinik.
}

Mit 3 Textabbildungen.

(Eingegangen am 8. März 1922.)

Die Entstehung von Carcinomen in epithelialen Hautcysten, die früher sogar vollständig geleugnet wurde, stellt ein recht seltenes Ereignis dar. Es kommt hierbei am häufigsten in einer bereits aufgebrochenen oder längere Zeit ulcerierten Cyste zu einer malignen Wucherung der Wand oder seltener zur Carcinomentwicklung in noch geschlossener Cyste. Gerade diese Fälle aber sind es, welche uns über die Vorgänge, die zur Entstehung des Carcinoms führen, aufklären können. Über einen hierher gehörenden Fall, den wir an unserer Klinik beobachten konnten, wollen wir daher im folgenden berichten.

Der 48 jährige Hilfsarbeiter F. V. bemerkte schon seit mehr als 10 Jahren an der AuBenseite des rechten Oberschenkels eine schmerzlose, gut verschiebliche Geschwulst, die ihm weiter keine Beschwerden verursachte. Seit Juni 1921 ist nun diese Geschwulst größer und schmerzhaft geworden, die Haut darüber gerötet. Vor einigen. Tagen ist sie aufgebrochen und entleerte dünnflüssigen Fiter. Pat. gibt an, daß er sich in letzter Zeit niemals an dieser Stelle verletzt hätte, nur seit die Geschwulst gewachsen sei, habe'er sich öfter daran gestoßen. Auch eine Verletzung vor längerer Zeit, bevor er die Geschwulst bemerkt habe, lehnt der Patient bestimmt ab.

Pat. gelangte am 27. IX. 1921 zur Aufnahme an unsere Klinik. Der senile, hagere Mann zeigt an der Außenseite des rechten Oberschenkels, knapp an der Glutäalfurche einen etwa hühnereigroßen, derb elastischen, unter der Haut gelegenen Tumor, der auf der Unterlage, der Fascia lata, nach allen Richtungen gut beweglich ist. Die Haut über dem Tumor ist lividrot verfärbt, allenthalben verschieblich und ist nur an zwei Stellen, an der Kuppe des Tumors und an der abhängigen Partie inniger mit diesem verwachsen. Dort ist die Haut in etwa Hellerstiuckgröße verdünnt, hellrot und es besteht deutlich Fluktuation, namentlich an der unteren Stelle, in deren Zentrum auch eine kleine Fistel mündet, die serösen Eiter entleert. In der nächsten Umgebung finden sich einige dilatierte Venen sowie mehrere braunrote Flecke, wie nach abgelaufenen Furunkeln. In der rechten Leistengegend mehrere haselnußgroße, derbe Drüsen.

Die am 28. IX. vorgenommene Probeexcision ergab ein unter der Haut gelegenes, mit dieser nirgends in Verbindung stehendes Plattenepithelcarcinom mit starker Verhornung und Hornperlenbildung, das anscheinend von einer unter der 
Haut gelegenen Epithelauskleidung einer Cyste ausgeht. Auf Grund dieses histologischen Befundes sowie der scharfen Abgrenzung des Tumors gegen die Unterlage und die Umgebung und der Verschieblichkeit der Haut über dem Tumor stellten wir die Wahrscheinlichkeitsdiagnose auf Carcinom in einem „Atherom" und überwiesen den Fall zwecks Radikaloperation der Chirurgischen Klinik Prof. Schloffer.

Dort wurde am 4. X. die Excision des Tumors 2-3 Querfinger im Gesunden mit Excision der darunter liegenden Fascie und eines Teiles der Muskulatur vorgenommen. Gleichzeitig wurden die vergrößerten rechtsseitigen Leistendrüsen exstirpiert.

Der Tumor stellt einen $5^{1 / 2} \mathrm{~cm}$ langen, $4 \mathrm{~cm}$ breiten und ca. $2 \mathrm{~cm}$ hohen unter der Hant gelegenen flachen Knoten dar, dem zwei etwa haselnuß- und kirschkerngroße Tochterknoten aufsitzen, die inniger mit der Haut verbunden sind, und von denen der erstere an seiner Kuppe ein über linsengroßes flaches Geschwür der bedeckenden Haut aufweist. Ein Schnitt durch die Kuppe des Tumors (Abb. 1) ergibt, $\mathrm{da} B$ es sich um eine cystische Geschwulst handelt. Nach oben zu (in der Abbildung rechts) ist das $\mathrm{Cy}$ stenlumen und die Wand anscheinend noch völlig normal. Das Lumen enthält abgestoßene Hornmassen und Detritus, die Wand wird von einem gelbweißen, ziemlich derben Gewebe gebildet, das

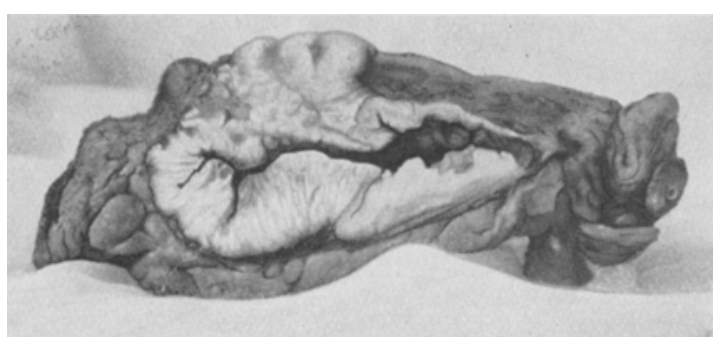

Abb. 1. gegen das Lumen mehrere papilläre Excrescenzen aufweist, nach außen aber eine scharfe feinzackige Grenzlinie zeigt. An diese schließt sich eine etwa 5 mm dicke Kapsel mit deutlicher Parallelstreifung an, die dort, wo sie der Haut benachbart ist, ohne scharfe Grenze in das subcutane Gewebe übergeht. Am unteren Pol dieser Cyste (in der Abbildung links) besteht ein Defekt der Cystenwand. Hier schlieBt sich ohne deutliche Abgrenzung ein etwa walnußgroßer Knoten von braungelber Farbe an, der eine ausgesprochene, meist radiär gestellte Felderung zeigt und von einem querverlaufenden Spalt durchzogen wird, der durch den beschriebenen Wanddefekt mit dem Lumen der Cyste in Verbindung steht. Auch dieser Knoten ist an seiner Basis und nach unten von einer schmalen, anscheinend bindegewebigen Kapsel umschlossen, während ihm gegen die bedeckende Haut zu zwei gleich gebaute Tochterknoten aufsitzen, von denen der erste bis an die hier verdünnte Haut heranreicht, aber noch immer scharf von ihr geschieden ist, während der zweite, in seinem oberen Teil nekrotisch, durch eine schmale Fistel mit dem oben beschriebenen flachen Geschwür der Haut zusammenhängt.

Aus der einen Hälfte des Tumors wurden mehrere Scheiben entnommen und histologisch untersucht. Ein Schnitt ungefähr durch die Mitte des Tumors zeigt das Bild einer unter der Haut gelegenen Cyste (Abb. 2). Ihre Wand wird oben von einer ziemlich breiten Schichte kernarmen Bindegewebes gebildet, dessen Bündel zum Teil parallel, zum Teil in spitzem Winkel sich kreuzend verlaufen und zwiscben denen größere und kleinere Gefäße liegen. Zwischen diesen Bindegewebsbündeln finden sich ziemlich reichlich meist um die Gefäße angeordnete kleinzellige Infiltrate. Nach innen zu gehen von diesem bindegewebigen Balg zahlreiche gut entwickelte Papillen ab, die mit Epithel ausgekleidet sind. Dieses zeigt alle Schichten der äußeren Haut, also basal eine Lage ron Cylinderzellen, 


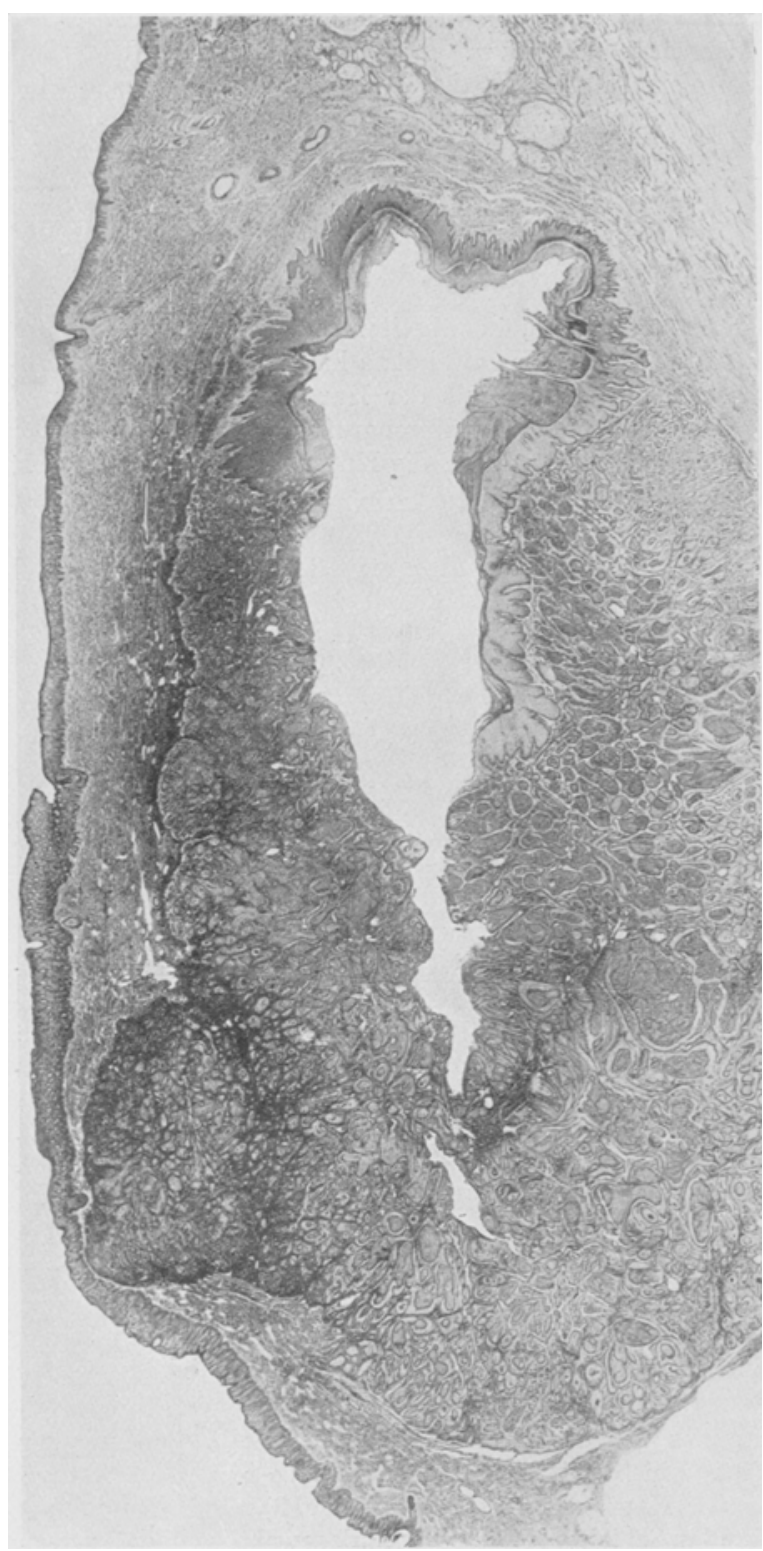

Abb. 2.

auf diese folgend eine mehrschichtige Lage von polygonalen Zelien, entsprechend dem Stratum spinosum, an dassich ein meist deutliches Stratum granulosum anschlie Bt, das in eine breitere oder schmälere, meist aber ziemlich mächtige Hornschichte übergeht. Von Anhangsgebilden der Haut finden sich nur einige wenige Haare in atrophischen Haarbälgen. Vereinzelt cieht man in der Hornschichte mit Hämatoxylin tiefblau gefärbte kleine Kalkschollen. Diese der äußeren Haut entsprechende Wandauskleidung nimmt aber nur etwa ein Drittel des Hohlraumes ein. An sie schliebt sich in zwei- bis vierfacher Wandstärke ein Gewebe an, das aus soliden Strängen und Nestern besteht, die sich aus Epithelzellen aufbauen und durch kleinzellig infiltrierte Bindegewebssepten geschieden werden. Diese Stränge lassen an der Peripherie eine deutliche Cylinderzellschichte erkennen, nach innen zu bestehen sie aus polygonalen Zellen und zeigen im Zentrum mehr oder weniger ausgedehnte, zwiebelschalenartig angeordnete, mit Eosin intensiv rotgefärbte Verhornungen und Hornperlen. Auch dieses Gewebe ist fast allseits von einer bindegewebigen Kapsel umsehlossen, gegen die äußere Haut zu finden sich aber vereinzelt derartig€ Züge auch schon in der Kapsel, und an einer Stelle haben sie diese durchbrochen und steigen 
hier bis gegen die äußere Haut auf, sind aber von dieser stets noch durch eine schmale Zone kleinzelliger Infiltration geschieden. Die äußere Haut selbst zeigt keine Besonderheiten.

In mehr gegen die Peripherie des Tumors geführten Schnitten (Abb. 3) sieht man die Cystenwand allseits von einer der äußeren Haut entsprechenden papillentragenden Epithelauskleidung gebildet, welche nach innen zu mächtige Verhornung aufweist. Anhangsgebilde wie Haare oder Haarbälge sind hier nicht nachweisbar. Nach außen zu wird diese Auskleidung ungefähr bis zur Hälfte von einer breiten bindegewebigen Kapsel umschlossen, während die andere Hälfte von den oben beschriebenen aus Epithelzellen aufgebanten Zügen und Nestern mantelartig

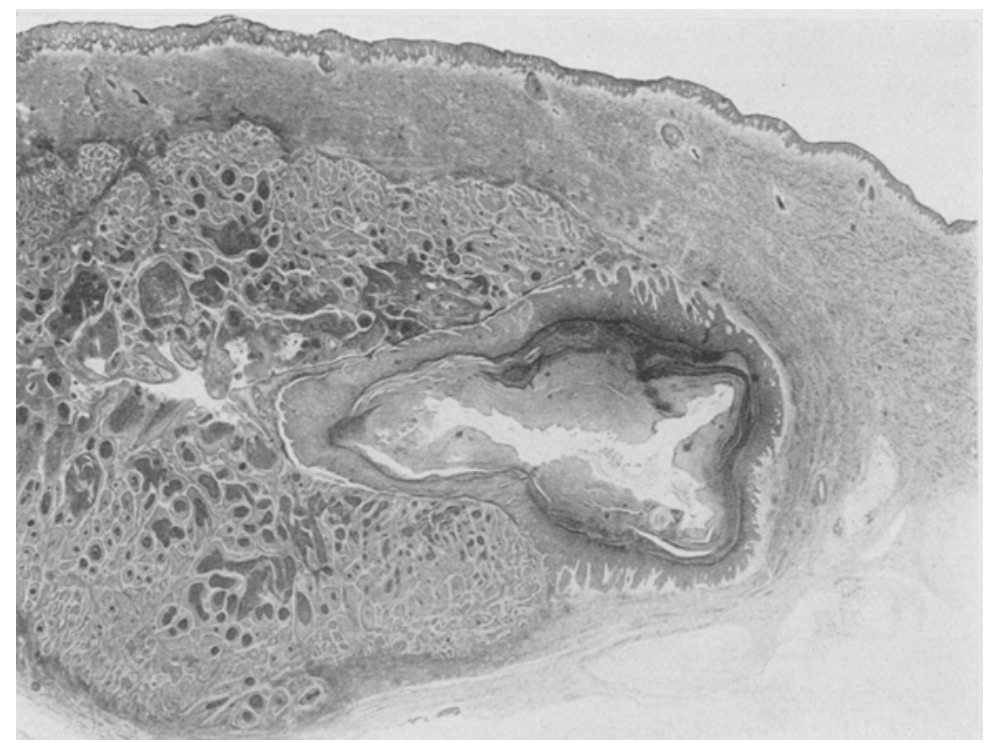

Abb. 3.

umgeben wird, so zwar, daß aber stets noch eine schmale entzündliche Zone zwischen ihnen und der Wand bleibt. Diese Tumormassen werden nach außen allseits von einem entzündlichen Infiltrate und einer breiteren oder schmäleren bindegewebigen Kapsel umgeben und erreichen hier nirgends die äußere Haut.

Erwähnt sei schließlich noch der Befund an der oben beschriebenen Fistel. Die vollkommen normale äußere Haut zeigt hier eine kleine Lücke. Unter der Haut, von dieser aber stets getrennt, finden sich wieder die schon beschriebenen Stränge und Nester des Tumorgewebes, welche sich nur im Bereiche des Defektes dicht an die Papillen der äuBeren Haut anlegen und die hier und im Bereiche der Fistel mehr oder weniger ausgedehnte Nekrosen zeigen.

Es handelt sich also nach dem histologischen Befund um eine in der Subcutis gelegene Dermoidcyste der Haut, die durch eine ziemlich breite bindegewebige Kapsel scharf gegen die Umgebung abgegrenzt ist. Von dieser gehen zahlreiche Papillen ab, welche mit einer gut entwickelten normalen Epidermis überkleidet sind und in den obersten Schichten starke Verhornung zeigen. Der Inhalt der Cyste besteht aus 
abgestoßenen Hornmassen und Detritus. Von Anhangsgebilden der Haut finden sich nur spärliche schlecht entwickelte Haare in atrophischen Haarbälgen. An einer Stelle der Wand, und zwar am unteren Pol der Cyste, ist es nun zur Entwicklung eines Plattenepithelcarcinoms mit reichlicher Hornperlenbildung gekommen. Dieses Carcinom ist nach außen - nicht gegen das Cystenlumen - gewachsen, hat zunächst die ganze Kapsel erfüllt, so zwar, daß es die seitlichen Teile der Cystenwand mantelartig umgibt, daß sich aber bis auf die Stelle, von der es ausgegangen ist, allenthalben ein breiterer oder schmälerer Zwischenraum zwischen Carcinom und Cystenwand nachweisen läßt. An einer Stelle hat der Tumor schließlich die Kapsel durchbrochen und ist hier gegen die äußere Haut zu gewachsen. Auch hier steht er aber nirgends mit dieser in Verbindung, sondern ist auch dort, wo er ganz an sie heranreicht, immer noch durch eine schmale Zone entzündlichen Gewebes von ihr getrennt, bis auf eine kleine Partie, wo nekrotische Teile des Tumors durch eine Fistel bis an die äußere Haut heranreichen. Was in unserem Falle die Veranlassung zur Entwicklung des Carcinoms gegeben hat, können wir nicht mit Bestimmtheit sagen, doch drängt sich uns, nach der Lage des Tumors und namentlich nach seinem Ausgange von der abhängigen Partie des Dermoids, der Gedanke auf, daß häufige Irritationen der Cyste beim Setzen usw. wohl eine Ursache für die Carcinomentwicklung gegeben haben.

Dermoide am Oberschenkel sind nicht häufig, doch wurden sie schon beschrieben. So beschreibt Chiari ein derartiges Dermoid, bei dem die von einer umschriebenen Stelle der Wand ausgehenden papillären Excrescenzen die gegenüberliegende Cystenwand und die deckende Haut durchbrochen haben und in Form eines Hauthornes nach außen gewachsen waren. Differentialdiagnostisch könnte man höchstens noch an eine traumatische Epithelcyste als Ausgangspunkt des Carcinoms denken. Dagegen spricht die Anamnese des Patienten, der jede Verletzung an dieser Stelle ablehnt, und der sich doch an ein stärkeres Trauma, denn nur ein solches könnte zur Epithelverlagerung und Cystenbildung geführt haben, erinnern müßte, sowie der Umstand, daß in der Wand der Cyste einzelne Haare nachgewiesen werden konnten, während nach postembryonaler Verlagerung von Hautkeimen nur Epidermoidcysten, nicht Dermoide entstehen (Lexer).

Bevor wir nun an die Erörterung der hierher gehörigen, bisher beschriebenen Fälle von Carcinombildung in epidermalen Hautcysten gehen, müssen wir kurz die Ätiologie und Anatomie der verschiedenen hierher gehörigen cystischen Geschwülste der Haut besprechen, um so mehr als namentlich in der älteren Literatur hier oft dieselben Bezeichnungen für verschiedene Bildungen vorkommen. Die in Frage kommen- 
den Gebilde sind das Atherom, das Epidermoid und Dermoid und die traumatischen Epithelcysten.

Namentlich der Ausdruck Atherom, den wir wohl nur mehr als klinischen Begriff führen können, hat hier viel Verwirrung angerichtet. Wir verstehen darunter erbsen- bis faustgroße, meist auf der Unterlage gut verschiebliche Tumoren von kugeliger Form, welche bald in der Tiefe des Coriums, bald im subcutanen Gewebe sitzen, deren Innenfläche mit Epidermiszellen ausgekleidet ist, und die meist einen grützebreiäbnlichen Inhalt von abgestoßenen Epithelien, Cholestearinkristallen, Detritusmassen und Fett zeigen. Nach den genauen Untersuchungen Chiaris haben wir es aber mit zweierlei genetisch scharf zu trennenden Gebilden zu tun, welche sich meist auch anatomisch und histologisch voneinander abgrenzen lassen. Es handelt sich zum Teil um sog. Retentionseysten der Haut, die bei Verlegung der Hautfollikel durch Retention des Inhalts aus den Haarbälgen und Talgdrüsenausführungsgängen entstehen (Follikelcysten Chiaris), zum Teil um Neubildungen meist cystischer Natur, die aller Wahrscheinlichkeit nach ihren Ursprung einer im fötalen Leben stattgehabten Abschnürung von in die Tiefe gedrungenen Epidermiszapfen verdanken (Epidermoide, Heschl, Franke). Erstere stellen meist kleine, höchstens bis nußgroße, die Talgdrüsenfollikelcysten wahrscheinlich nur hanfkorngroße, teils cutan, teils subcutan gelegene Cysten dar, die eine ganz zarte, vollkommen glatte, $d$. h. ganz papillenlose Wand besitzen und deren Innenauskleidung aus einer $\mathbf{2 - 4}$ schichtigen Lage abgeplatteter Epidermiszellen besteht. Bei genauer Untersuchung läßt sich bei ihnen meist noch der mehr oder weniger verödete oder verstopfte Ausführungsgang und als Anhang der unteren oder seitlichen Cystenwand meist noch der verödete Haarbalggrund oder Reste der Talgdrüsen nachweisen. Sie finden sich an allen Stellen des Körpers, wo Haarbälge oder Talgdrüsen vorhanden sind. Die Cysten der zweiten Art sind meist größer, liegen immer subcutan, wobei sie sich oft in beträchtliche Tiefe erstrecken, ihre bindegewebige Wand erscheint durchwegs dicker, zeigt mehr oder weniger, meist aber gut ausgebildete Papillen und ihr Epithel zeigt den typischen Bau der vollständig entwickelten Epidermis. Die kleinsten von ihnen sind oft fast solid und lassen nur mikroskopisch einen Hohlraum erkennen. Ein Ausführungsgang, resp. eine Kommunikation mit der Oberfläche ist meist nicht vorhanden, kann aber vorkommen. Sie liegen immer fissural, d.h. im Bereiche oder der Nachbarschaft der einstigen Schlußlinien der Körperhöhlen beziehungsweise sonstiger embryonaler Schlußlinien, wie sie z. B. bei der Digitation an der Hand oder beim Herauswachsen der Extremitäten sich bilden (Chiari). Dementsprechend finden sie sich am häufigsten in der Mittellinie des Kopfes, über den Fontanellen, an der Glabella, der Regio supraorbitalis, Wange 
Nase und Umgebung des Ohres, Plica nasolabialis, Regio frontalis und an der Vorderseite des Halses, seltener in der Mittellinie des Sternums, der Nabelgegend, in der Kreuzbeingegend, in der Palma manus, der Regio deltoidea und lumbalis sowie am Oberschenkel. Sie sind stets angeboren, kommen meist schon in frühester Kindheit zur Beobachtung, können aber auch erst später sich vergrößern und dadurch dem Träger auffallen.

An die Epidermoide schließen sich nach ihrer Genese und ihrem anatomischen Bau unmittelbar die Dermoide der Haut an, mehr oder weniger cystische Bildungen, welche gleichfalls embryonalen Abschnürungen, und zwar Resten der Kiemengänge oder Inklusionen von Haut oder epithelialen Anteilen in der Thefe des Coriums oder des subcutanen Gewebes ihren Ursprung verdanken (Kaufmann), deren Wand aber den vollständigen Bau der äußeren Haut mit den verschiedenen Anhangsgebilden wie Haar-, Talg- und Schweißdrüsen aufweist. Auch sie liegen, entsprechend ihrer Genese, stets fissural. Die gleiche Genese der Epidermoide und Dermoide sowie die Ähnlichkeit in ihrem anatomischen Bau, indem die Wand der ersteren Cutisstruktur, aber ohne die verschiedenen Anhangsgebilde, die Wand der letzteren daneben auch die Anhangsgebilde in bald stärkerer, bald schwächerer Entwicklung zeigt, sowie der Umstand, daß sich zwischen beiden Cysten alle Übergänge finden, veranlaßte Chiari, sie in eine Gruppe einzuteilen.

Von den Dermoiden der Haut vollständig zu trennen sind die sog. Dermoide des Ovars, gleichfalls Cysten, deren Innenauskleidung Cutisstruktur zeigt, die aber in ihrer Wand stets Teile aller drei Keimblätter aufweisen, so daß sie wohl heute allgemein den Embryomen zugerechnet werden und nicht in den Kreis unserer Erwägungen fallen.

Schließlich gehören hierher noch die traumatischen Epidermoidcysten, Implantationscysten, resp. Epithelcysten nach Garrè, welche analog den experimentell erzeugten Cysten durch Implantation von Epidermis oder Epidermis und Mutterboden in die Tiefe des Coriums oder der Subcutis entstehen, und die gewöhnlich an der Volarseite der Hand sitzen. Sie gleichen in ihrem Bau den Epidermoiden.

Carcinomentwicklung in derartigen Cysten der Haut ist nun, soweit wir die Literatur verfolgen konnten, bisher in 38 Fällen beschrieben worden. In der Mehrzahl handelt es sich nach der Beschreibung um Carcinome in „Atheromen“ oder auf deren Boden, und da gerade die letzte hierher gehörige Arbeit von Kurtzahn diese Fälle wieder von den Fällen von Carcinom auf dem Boden von Dermoiden und Epidermoiden abtrennt, halten wir es nach dem oben Gesagten für notwendig, zu untersuchen, inwieweit die hierher gehörigen Beobachtungen den Retentionscystenatheromen resp. den Neubildungsatheromen zugehören.

Franke gab 1887 eine genaue Zusammenstellung von 18 Fällen von 
„Atheromearcinomen", welche bis auf das Jahr 1804 zurückgeht. Soweit wir die dort zitierte Literatur zurückverfolgen konnten, hat sie uns keine näheren Aufschlüsse über die Art der Atherome gebracht, so daß wir uns im wesentlichen den Ansichten Frankes anschließen müssen.

Es handelt sich in 8 Fällen um isolierte Atherome des Schädels, nur in 2 Fällen (Lücke und Weichselbaum) bestanden mehrere Atherome, von denen eines ,,carcino-

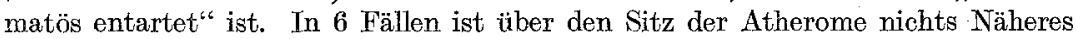
angegeben und in 2 Fällen (Abenethy und Völker) sind es Atherome, welche an der Nase bzw. zwischen den Schulterblättern saßen, die zum Carcinom geführt haben. In allen diesen Fällen handelte es sich, soweit überhaupt anamnestische Daten existieren, um eine meist längere Zeit bestehende, auf mechanischem oder chemischem Wege erzeugte Reizung schon ulcerierter Atherome, welche hier zur ,carcinomatösen Entartung" geführt hat, zum Teil bestand dieser chronische Reiz noch nach Exstirpation der Geschwiulste und hat erst Jahre nachher zur Entwicklung eines Carcinoms geführt, dessen Ursprụng in zurückgebliebenen Teilen des Balges angenommen wird.

Entsprechend der Ansicht Frankes über die Entstehung des Atheroms als Epidermoid, faßt er auch die Atheromcarcinome als maligne Wucherungen auf, die von embryonal versprengten Keimen ausgehen.

Anzureihen wäre bier noch ein Fall Riedels, in dem sich bei einer 51 jährigen Patientin aus einer Atheromeyste des rechten Oberschenkels, die seit 20 Jahren bestand und vor $3 / 4$ Jahren perforiert war, ein Plattenepithelcarcinom entwickelt hat.

Für unsere Ausführungen sind nun die seit Franke beobachteten Fälle, bei denen uns meist eine ausführliche histologische Beschreibung zur Verfügung steht, wichtiger und deshalb müssen wir sie kurz referieren.

1890 beschreibt Franke selbst zwei carcinomatös entartete Epidermoide des Daumenballens.

Bei einem 40 jährigen Mann bestehen seit 3 Jahren am Daumenballen der rechten Hand 2 kleine Epithelcysten, deren Auskleidung vollständig der Epidermis entspricht und die auch einzelne Papillen aufweisen. In einem Teil der Wand ist es zur Umwandlung in ein alveoläres Carcinom ohne Bildung von Hornperlen gekommen, das auch auf die bindegewebige Kapsel übergreift. Außer einem Schlage gegen eine Türkante wird jede Verletzung negiert. Franke nimmt an, daB diese Epidermoide aus fötal abgesprengten Keimen entstanden sind und widerlegt für seinen Fall die Möglichkeit einer traumatischen Entstehung.

Es ist dies anscheinend der erste Fall in der Literatur, wo Carcinomentwicklung in einem geschlossenen Epidermoid beschrieben wird.

Der nächste Fall stammt von Boldt und betrifft ein Plattenepithelcarcinom in einem ,Atherom".

Bei einer 68 jährigen Frau bestanden seit 30 Jahren mehrere Atherome, von denen eines vor 3 Jahren verietzt wurde, aufbrach und seither fistelte. Es entstand eine pflaumengroße, etwas platte Geschwulst, deren Hauptmasse pilzartig die Haut um $1 \mathrm{~cm}$ überragte. Der Tumor hat das Aussehen eines papillären Carcinoms. Krische hält die Cyste für ein Dermoid, und zwar auf Grund der histologischen Beschreibung: ,Normale Epidermis und Corium nur noch teilweise erhalten. Das Corium weist in dieser Partie seine typischen Attribute auf, also Haare mit 
Papillen, Wurzelscheiden, Haarbalg, Talgdrüsen und Muskel, ferner Schweißdrüsenknäuel und Blutgefäße." Auen Ribbert zählt den Fall zu den Carcinomen in Dermoiden.

Kurtzahn nimmt an, daß es sich um ein Atherom handelte, indem er die zitierte Stelle nicht auf die Cystenwand, sondern auf die äuBere Haut bezieht, und zwar nimmt er anscheinend ein Retentionscystenatherom an, weil er in seinen Ausführungen diesen Fall aus den Mitteilungen von Carcinombildung in Dermoiden und Epidermoiden ausscheidet. Uns selbst war die Originalarbeit nicht zugänglich.

Zwei weitere Fälle beschreibt $H$. Wolff, bei denen es sich beide Male um Carcinombildung in Dermoiden resp. auf deren Boden bei jugend. lichen Individuen handelt.

Seine erste Beobachtıng betrifft einen 21 jährigen Mann, der am linken Augenwinkel eine walnußgroße Geschwulst trägt. Nach der Exstirpation zeigt sich das typische Bild eines Dermoids, in dem es in ca. $1 / 10$ der Cystenwand zur Wucherung eines malignen Tumors vom Charakter des Krompecherschen Tumors - alveoläres Cylinderepithelcarcinom ohne jede Tendenz zu konzentrischer Schichtung, Verhornung oder Bildung von Perlkugeln - kam. Auch hier erfolgte die Carcinomentwicklung in geschlossener Cyste. Die Ausbreitung erfolgte nicht gegen das Cystenlumen, sondern subepithelial innerhalb der Kapsel.

Sein nächster Fall betrifft ein 21 jähriges Mädchen, welches eine dem Kreuzbein aufsitzende, hühnereigroße Dermoidcyste aufweist. Nach mehr als einem Jahre zeigten sich an der Stelle der exstirpierten Geschwulst 2 Fisteln, nachdem vorher eine entzïndliche Anschwellung an dieser Stelle incidiert worden war. Die Exkochleation der Fisteln ergab den Befund eines Plattenepithelcarcinoms mit Verhornung und Perlkugelschichtung, von dem Wolff annimmt, daß es aus Resten der Dermoidwand hervorgegangen sei, die bei der Exstirpation zurückgeblieben sind.

Mertens berichtet über zwei subcutan an der linken Halsseite sitzende allseitig geschlossene Dermoidcysten bei einem 47 jährigen Mann, von denen eine ,carcinomatös degeneriert" war.

Gleichzeitig bestand ein nach dem Oesophagus zu ulceriertes Plattenepithelcarcinom an der linken Seite des Kehlkopfes, das Verf. gleichfalls als eine carcino. matös entartete dritte Dermoidcyste auffaßt. Das in der Cyste entstandene Carcinom zeigt gegen das Lumen gerichtete zottenartige Auswüchse, die aus mehr kubischen und platten Zellen mit deutlicher Schichtung, aber ohne Perlkugelbildung bestehen. In einer Lymphdrüse der linken Halsseite sitzt eine Carcinommetastase.

Von Wichtigkeit ist der folgende von Krische beobachtete Fall von primären Krompecherschen drüsenartigen Oberflächenepithelkrebs in geschlossenem ,Atherom".

Die Erkrankung betriff̂t einen 40 jährigen Mann, der vor einem Jahre in der linken Lende einen ca. haselnußgroßen, schmerzlosen Knoten bemerkte, der sich ziemlich rasch zu einem taubeneigroßen Tumor entwickelte. Nach der vorliegenden genauen Beschreibung haben wir diesen Fall von dem Atherom im engsten Sinne, d. h. Retentionseystenatherom, abzutrennen und den Neubildungsatheromen, d. h. den Dermoiden resp. Epidermoiden, zuzurechnen. Dies ergibt sich aus der histologischen Beschreibung der Wand der Cyste: „Das Epithel zeigt, wie schon gesagt, keine Papillen. Es folgen auf eine zylindrische Schicht (der basalen Zellschicht des Stratum Malpighii entsprechend) mehrere Schichten polygonaler Zellen, wie die des Stratum spinosum der Epidermis, darüber langgestreckte Zellen, denen des Stratum granulosum vergleichbar, dann verhornte, ganz abgeflachte 
Zellen. Es fehlt also am Bau der Epidermis nur die papilläre Anordnung des Stratum Malpighii. Die beigegebene Abbildung zeigt außerdem schön entwickelte Papillen mit in diese hereinziehenden Gefäßen, so daß das Fehlen der Papillen, das Verfasser mehrfach hervorhebt, auf einem Irrtum zu beruhen scheint.

Wir haben es also mit einer Epidermoidcyste zu tun, deren Wand eine typische Epidermis mit Papillen darstellt. Das Carcinom hat sich hier in Form von papillösen Wucherungen gegen das Lumen zu entwickelt.

In derselben Arbeit berichtet Krische über einen von Garrè beobachteten Fall.

Bei einem 63 jährigen Mann ist eine seit mehr als 10 Jahren bestehende, ca. nußgroße ,Haarbalggeschwulst" wahrscheinlich infolge Verletzung beim Kämmen aufgebrochen und fistelt. Im Laufe des letzten Jahres entwickelte sich an dieser Stelle langsam ein Geschwür, dessen histologische Untersuchung den Befund eines Cancroids ergibt.

Nähere Angaben über den. Bau der Cystenwand fehlen.

In einem Zeitraum von 2 Jahren beobachtete Linser an der Tübinger Chirurgischen Klinik 10 resp. 8 Fälle von Epitheliomen und Carcinomen in Dermoiden oder Fpidermoiden und zieht daraus den Schluß, daß derartige Fälle nicht gar so selten, wie man gemeinhin annimmt, vorkommen.

Seine Fälle betreffen durchwegs ältere Personen zwischen 54 und 65 Jahren, bei denen in mehr als der Hälfte die Geschwülste seit frühester Jugend bestanden. In 5 Fällen (Fall I, II, IV, V, VI) handelt es sich um Tumoren des Schädels, welche mit Ausnahme des letzten Falles, wo daneben zahlreiche „Atherome" beschrieben werden, stets isoliert saßen. In einem Fall (III) saß der Tumor an der Außenseite des linken Oberschenkels über dem Kniegelenk, im Fall VIII in der Mitte des rechten Orbitalrandes und Fall VII im rechten vorderen Gaumensegel. Alle diese Tumoren liegen subcutan, sind meist gut abgekapselt und ohne Verbindung mit der Epidermis oder höchstens in sekundürer Verbindung mit dieser.

Der erste Fall betrifft eine Dermoidcyste, in der sich wahrsoheinlich nach einem Trauma eine papillomatöse Wucherung mit anscheinendem Übergang in ein Carcinom entwickelt hat. Die Entwicklung erfolgte hier wie in den späteren Fällen in geschlossener Cyste. Fall II und III stellen beide subcutane, gut abgekapselte Epitheliome dar, welche in einem bindegewebigen Balg Nester und Stränge von geschichtetem, zum Teil verhorntem Plattenepithel zeigen. In beiden Fällen findet sich in größerem oder geringerem Maße Verkalkung. Der Verfasser nimmt an, daß es sich hier um Epidermoide als Ausgangspunkt der Tumorbildung handelt, da vereinzelt noch kurze Strecken eines. Epithelbelags nachweisbar waren und nimmt an, daß hier ähnliche Papillome, wie in Fall I, den ganzen Hohlraum erfüllt haben und deren Zotten allseits mit der Cystenwand verwachsen sind.

Der IV. Fall betrifft einen hühnereigroßen Tumor, der sich aus cystischen und soliden Anteilen aufbaut. Erstere zeigen eine Auskleidung von papillär. angeordnetem Epithel, letztere bestehen aus Epithelnestern und Strängen mit Cancroidperlen. Dazwischen finden sich bald mehr, bald weniger gut erhaltene Talgdrüsen. Es wird hier, auf Grund der Epithelauskleidung und des Befundes von Talgdrüsen, ein Dermoid als Ausgang angenommen.

Fall $\vec{V}$ betrifft einen ausgedehnten, größtenteils ulcerierten flachen Tumor an der linken Kopfseite einer 56 jährigen Frau, der sich aus einem seit der Kindheit bestehenden, kleinen Knötchen über dem Ohr entwickelt hat. Der Tumor, der sich 
aus einzelnen, durch bindegewebige Kapseln geschiedenen Knoten aufbaut, setzt sich zum Teil aus drüsigen Bildungen, mit meist einschichtigem hohem Cylinderepi: thel und weitem Lumen, zum Teil aus Nestern epitheloider Zellen mit Epithelzwiebeln zusammen. Nur an den Randpartien des Tumors und in der einstigen Kapsel findet man Züge und Nester kleiner, polygonaler Krebszellen, nach Art des Carcinoma scirrhosum. Vereinzelt sieht man zwischen den Drüsenschläuchen große Epithelzellen von kubischer oder polygonaler Form, die Linser als Talgdrüsenelemente deutet.

Der nächste Fall betrifft ein etwa taubeneigroßes Atherom (Epidermoid ohne Haare) über dem Proc. mastoideus, von dessen Wand ein Plattenepithelcarcinom, das sehr zu Nekrosen neigt und reichlich Verhornung und Epithelperlen zeigt, ausgeht, das den größten Teil des Hohlraumes einnimmt, an einer Stelle die Wand durchbrochen hat und hier auf die bedeckende Haut übergreift.

Fall VII und VIII stellen schließlich kugelige Tumoren dar, die von einer mehr oder weniger erhaltenen bindegewebigen Kapsel umschlossen sind und zwar alveolär gebaute Carcinome, welche allenthalben auf die Umgebung, besonders auf die deckende Haut bzw. Schleimhaut übergreifen. Nur vereinzelte Epithelzwiebeln sprechen mit Wahrscheinlichkeit für den Ausgang dieser Tumoren von geschichtetem Pflasterepithel. Aus der Lage beider Tumoren, Fall VII im vorderen Gaumensegel, Fall VIII in der Mitte des Orbitalrandes und aus ihrem Hervorgehen aus schon lange bestehenden kleinen Knoten, schließt Linser, daß es sich auch hier um Carcinome handelt, welche aus embryonal versprengten Keimen, wahrscheinlich Epidermoiden, entstanden seien.

Soweit wir der histologischen Beschreibung folgen konnten - Abbildungen der Präparate fehlen - scheint uns der fünfte Fall Linsers, von dem auch er angibt, daß ihm seine Deutung große Schwierigkeiten bereitet hätte, nicht hierher zu gehören. Im Falle I und VI handelt es sich beide Male um eine cystische Geschwulst, I ein Dermoid, VI ein Epidermoid, bei denen nur das Carcinom im Falle I vielleicht in Zweifel zu ziehen ist und es möglich wäre, daß es sich um ein einfaches Papillom handelt, wie sie öfter in Atheromen beobachtet wurden. Die Fälle II und III betreffen Epitheliome, bei denen wohl der Ursprung aus epidermalen Hautcysten sichergestellt erscheint, die Natur der Cyste als Epidermoid aber nicht mehr sicher bewiesen werden kann. Im Falle IV scheint es sich um ein Dermoid zu handeln, das größtenteils in Carcinombildung aufgegangen ist. Die beiden letzten Fälle schließlich lassen unserer Ansicht nach einen zwingenden Schluß auf den Ursprung der Carcinomentwicklung nicht zu.

Krebsige Entartung von Kopfatheromen beschreibt Zesas.

Bei einer 68 jährigen Frau kam es im Anschluß an Verletzungen beim Kämmen zur Ulceration und Zerfall mehrerer Atherome des Schädels, die schon seit mehr als 20 Jahren bestanden haben sollen. Es entwickelte sich im Laufe von 5 Jahren ein carcinomatöses Geschwür, welches die linke hintere Hälfte der Kopfhaut einnimmt.

Eine genauere histologische Beschreibung liegt nicht vor.

Als nächster schildert Kaufmann die Entwicklung eines Carcinoms in einer geschlossenen epidermoidalen Cyste in der Tiefe der Mamma. 
Bei der 45jährigen Frau bestand neben der erwähnten hühnereigroßen Cyste ein Carcinoma simplex der Mamma.

Crone erwähnt gleichfalls einen Fall von Carcinom, das sich auf dem Boden eines ulcerierten Atheroms des Kopfes entwickelte. Auch hier fehlt die histologische Beschreibung.

Schließlich schildert Kurtzahn ein Plattenepithelcarcinom des Schädels mit reichlichen Krebsperlen und Nekrosen bei einer 69 jährigen Frau, das von der Wand eines Epidermoids seinen Ausgang genommen hat.

Von der ehemaligen Cyste war nur mehr die Wand als solche zu erkennen. Der Sitz in der Mittellinie des Kopfes über der kleinen Fontanelle sowie die anamnestische Angabe, daß seit frühester Jugend an dieser Stelle ein kleiner Knoten saß, der stets beim Kämmen hinderlich gewesen sei, sprechen für eine Entstehung aus einem Epidermoid.

Von den seit Franke beschriebenen 18 Fällen - den 5. Fall Linsers müssen wir nach dem oben Gesagten ausscheiden - können wir für unsere Untersuchungen bezüglich der Beteiligung der einzelnen Arten der epithelialen Hautcysten an der Carcinomentwicklung nur 15 Fälle verwerten, da in den restlichen Beobachtungen (Garrè, Zesas, Crone) eine genaue Beschreibung, namentlich aber ein histologischer Befund fehlt. Für diese Frage kommen in erster Linie jene Fälle in Betracht, bei denen es in noch geschlossener Cyste zum Wachstum des Carcinoms. gekommen ist und die Cystenwand noch so weit erhalten war, daß eine genaue Bestimmün der Cyste möglich ist. Hierher gehören 7 Fälle, von denen 3 auf das Dermoid entfallen (Wolff Fall I, Mertens, Linser Fall I) und 4 auf das Epidermoid (Franke, Krische, Linser Fall VI, Kaufmann). Nicht ganz sicher in Hinsicht auf die Carcinomnatur ist hierbei, wie schon oben erwähnt, der erste Fall Linsers, bei dem vielleicht ein einfaches Papillom vorliegt. Diesen Fällen ist als achter unsere Beobachtung von Carcinom in einem Dermoid anzureihen. Wir möchten hierbei nochmals betonen, daß es, wie schon Chiari hervorhebt, zwischen Dermoid und Epidermoid alle möglichen Übergänge gibt, so daß namentlich dort, wo nicht die ganze Cyste histologisch untersucht wurde - und soweit wir die Literatur verfolgen konnten, ist dies nur in den seltensten Fällen geschehen - sehr leicht die Möglichkeit besteht, daß aus dem Fehlen der Anhangsgebilde der Haut der niedrigere Typus, das Epidermoid, angenommen wird. Sehr lehrreich ist in dieser Beziehung auch unser Fall, denn auch hier war nur der Befund einiger weniger Haare in einzelnen Schnitten für die Diagnose Dermoid entscheidend, während meist nur eine gut entwickelte und stark verhornte, papillentragende Epidermis als Wandauskleidung vorlag, so da $\beta$ wir nach diesem Befund auf ein Epidermoid hätten schließen müssen. Es ist nun klar, daß die Klassifizierung der Cysten um so schwerer wird, je größere Teile der Wand in das Tumorgewebe aufgegangen sind. Hier wird sich viel- 
leicht zwischen Dermoid und Epidermoid kein Unterschied machen lassen und wir werden oft auch genötigt sein, mehr klinische Befunde, wie den Sitz des Tumors und die Anamnese zur Abtrennung dieser Tumoren von solchen, die aus Retentionseysten hervorgegangen sind, heranzuziehen. Danach haben wir von den mehr oder weniger soliden Tumoren vier zu den auf embryonale Keimversprengung zurückgeführten Cysten zu rechnen, und zwar einen zu den Dermoiden (Linser Fall IV) und drei zu den Epidermoiden (Linser Fall II und III, Kurtzahn). Bei völliger Zerstörung der Wand erscheint schließlich eine genaue Einteilung unmöglich, wie in den beiden letzten Füllen Linsers, von denen. er nach dem Sitz im Gaumensegel und in der Mitte des Orbitalrandes annimmt, daß es sich hier gleichfalls um Tumoren auf der Basis von Dermoiden resp. Epidermoiden handelt. Nur in zwei von den in Frage kommenden 15 Fällen haben wir es mit Carcinomen in bereits exulcerierter Cyste bzw. auf deren Boden zu tun. Von diesen müssen wir den einen Fall sicher den Dermoiden zuzählen (Wolff Fall II), ebenso wahrscheinlich den Fall Boldt, der zwar als Atherom beschrieben und von Kurtzahn diesen zugezählt, von Krische und Ribbert aber zu den Dermoiden gerechnet wird, eine Ansicht, der auch wir uns, soweit wir uns aus den knappen Referaten ein Bild machen konnten, anschließen möchten. Wir können uns nun recht gut vorstellen, daß bei derartigen exulcerierten Zysten die Diagnose oft recht schwer, wenn nicht unmöglich wird, namentlich dann, wenn das Carcinom auf die äußere Haut übergegriffen hat und nur mehr kleine Teile der Cystenwand erhalten geblieben sind. Es wird dann ein carcinomatöses Geschwür resultieren wie z. B. im Falle Zesas, und nur eine genaue Anamnese wird in einem Teil dieser Fälle das Hervorgehen des Tumors aus einer Epithelcyste der Haut erhärten können.

Die oben angeführte Zusammenstellung zeigt uns nun, daß in allen diesen Fällen zum Teil mit Sicherheit, zum Teil mit größter Wahrscheinlichkeit als Boden des Tumors Dermoide resp. Epidermoide der Haut in Frage kommen, jedenfalls Gebilde, welche auf eine embryonale Absprengung von Hautteilen zurückzuführen sind. In keinem Falle, vielleicht den Fall Boldt ausgenommen, läßt sich der Tumor mit Sicherheit auf eine Retentionscyste zurückführen. Wenn wir nun auch nicht behaupten können, daß in letzteren eine Carcinomentwicklung unmöglich ist, so spricht doch unsere Zusammenstellung dafür, daß, wenigstens was die Hauteysten anlangt, es im Sinne Ribberts die embryonal versprengte Keimanlage ist, welche den Boden für die maligne Wucherung abgibt. Daß bisher die Entstehung derartiger Carcinome aus Dermoiden für das Seltenere angenommen wurde, entspricht wohl hauptsächlich dem schlechten Sprachgebrauch des Wortes Atherom, unter dem wir uns doch meist auch heute noch eine Retentionscyste physiologischer 
Hautfollikel vorstellen. Viel zu dieser Ansicht hat die oft zitierte $\mathrm{Zu}$ sammenstellung Frankes über 18 ,Atheromcarcinome" beigetragen, obwohl gerade Franke in recht temperamentvoller Weise für die Abstammung der meisten Atherome aus versprengten Hautkeimen eintritt und auch an Stelle des Namens Atherom den Ausdruck Epidermoid von. Heschl übernommen und in die Literatur eingeführt hat.

Weitaus die größte Zahl der in Epithelcysten beobachteten Carcinome waren verhornende Plattenepithelkrebse mit reichlicher Hornperlenbildung, nur in 2 Fällen (Wolff Fall $\mathrm{I}$, und Krische) lag ein sog. Krompecherscher Tumor vor. Übergänge zum Alveolarcarcinom zeigen 2 Fälle von Linser. Auch unser Fall betrifft ein Plattenepithelcarcinom mit starker Verhornung und Perlkugelschichtung.

Solange wir es noch mit nicht zu sehr vorgeschrittenen Tumoren zu tun haben, läßt sich nachweisen, daß die Wucherung niemals in der ganzen Wand auf einmal erfolgt, sondern daß es immer einzelne Wandstellen sind, von denen das Carcinom ausgeht und bald in das Lumen der Cyste, bald gegen die Kapsel zu, diese erfüllend und schließlich durchbrechend, wächst.

Schon Franke hat hauptsächlich wohl aus den anamnestischen Daten, die seiner Zusammenstellung zugrunde liegen, angenommen, daß äußere Reize mechanischer und chemischer Natur zunächst zur Ulceration und Perforation des ,Atheroms" und schließlich zum Carcinom führen, wobei er diese Reize allerdings nur als wesentliches Glied in der Kette der verschiedenen Gelegenheitsursachen, welche die Krebsentwicklung bewirken, gewertet haben will. In den folgenden Beobach. tungen finden wir immer wieder den äußeren Reiz als eine der Ursachen der Carcinomentwicklung bald nur anamnestisch, bald anatomisch, indem das Carcinom sich meist an der Stelle der Cyste entwickelt, welche äußeren Schädigungen am nächsten liegt. Eine Ausnahme bilden hier nur der Fall Franke und Krische. Auch in unserer Beobachtung ist es auffallend, daß sich das Carcinom an der Stelle entwickelt hat, welche nach der Lage der primär vorgelegenen Cyste am ehesten ständigen Irritationen ausgesetzt ist, so daß auch wir den äußeren Reiz als einen mächtigen Faktor für die Carcinombildung betrachten müssen.

Meist sind es ältere Personen jenseits des fünften Lebensjahrzehntes, welche derartige Carcinome zeigen. Es entspricht dies unserer Erfahrung bezüglich der Hautkrebse, welche gleichfalls nach dem 40. Jahre relativ häufiger vorkommen. Die auffallende Tatsache, daß namentlich bei den aus Geschwülsten der Kopfhaut hervorgegangenen Tumoren die Träger meist dem weiblichen Geschlecht angehören, läßt sich vielleicht dadurch erklären, daß hier die Geschwülste unter den Haaren verdeckt sind und infolgedessen die Trägerinnen nicht so leicht zur frühzeitigen Entfernung der Geschwulst veranlassen. 
402 S. Schoenhof: Carcinomentwicklung in einem Dermoid der Haut.

Zusammenfassend können wir sagen, daß nur in seltenen Fällen von Epithelcysten der Haut Carcinome ausgehen. Diese entwickeln sich mit Vorliebe, wenn nicht ausschließlich, in Dermoiden und Epidermoiden, Gebilden, die auf embryonal verlagerte Hautteile zurückzuführen sind. Die Entwicklung erfolgt meist in höherem Alter, gewöhnlich wohl unter dem Einfluß mechanischer oder chemischer Irritationen.

\section{Literatur.}

Crone, Die Dermoidfisteln über dem Steißbein. Münch. med. Wochenschr. 1917, Nr. 16. - Chiari, Über die Genese der sog. Atheromeysten der Haut und des Unterhautzellgewebes. Zeitschr. f. Heilkunde 12. 1891. - Franke, Über das Atherom, besonders mit Bezug auf seine Entstehung (das Epidermoid). Arch. f. klin. Chirurg. 34. - Franke, Beiträge zur Geschwulstlehre. Virchows Arch. f. pathol. Anat. u. Physiol. 121. 1890. - Kaufmann, U̇ber Enkatarrhaphie von Epithel. Virchows Arch. f. pathol. Anat. u. Physiol. 97. 1884. - Kaufmann, Lehrbuch der speziellen pathologischen Anatomie S. 1098. 1911. - Krische, Ein Fall von primärem Krompecherschen drüsenartigem Oberflächenepithelkrebs in geschlossenem Atherom. Beitr. z. klin. Chirurg. 31. 1901. - Kurtzahn, Über die carcinomatöse Degeneration der Epithelcysten der Haut usw. Virchows Arch. f. pathol. Anat. u. Physiol. 230. 1921. - Linser, Über die Entwicklung von Epitheliomen und Carcinomen in Dermoideysten. Beitr. z. klin. Chirurg. 31. 1901. - Mertens, Carcinom auf dem Boden eines Dermoids. Beitr. z. klin. Chirurg. 31. 1901. Ribbert, Das Carcinom des Menschen. 19I1. - Riedel, Dtsch. Zeitschr. f. Chirurg. 15, 92. - Wolff, Carcinom auf dem Boden des Dermoids. Arch. f. klin. Chirurg. 62. 1900. - Zesas, Über die krebsige Entartung der Kopfatherome. Münch. med. Wochenschr. 1904, Nr. 37. - (Weitere Literatur bei Franke und Kurtzahn.) 